\title{
Notch1 intracellular domain increases cytoplasmic EZH2 levels during early megakaryopoiesis
}

\author{
A Roy, NP Basak and S Banerjee
}

Notch pathway is a well-known factor in the development of lymphoid lineage. However, its role in the myeloid lineage has remained ambiguous. We looked into the effect of Notch1 on the megakaryocytic lineage commitment and found an increase in megakaryocyte-specific lineage markers upon transfection with Notch1 intracellular domain (NICD). This effect was mediated by Akt whereby constitutive activation of Akt increased the megakaryocyte markers, whereas inhibition of Akt signalling reduced these marker levels. Along with the change in differentiation status, NICD-induced initiation of early megakaryopoiesis was accompanied by an increased cytoplasmic enhancer of zeste homolog-2 (EZH2) expression. This process was found to be Akt-dependent, and inhibition or overexpression of Akt lead to concurrent changes in EZH2 levels. To elucidate the function of EZH2 in the cytoplasm, novel cytoplasmic interactors of EZH2 were identified by co-immunoprecipitation followed by matrixassisted laser desorption ionization MS/MS-based protein identification, and thus, PDIA1 and LIM domain kinase-1 (LIMK1) were identified. Interaction of EZH2 with LIMK1 changed the activity of cofilin (a downstream target of LIMK1) towards actin filaments, thereby leading to lower filamentous actin content within these cells. Thus, Notch1 not only induces early megakaryopoiesis but also prepares these cells for subsequent morphological changes.

Cell Death and Disease (2012) 3, e380; doi:10.1038/cddis.2012.119; published online 23 August 2012

Subject Category: Experimental Medicine

Notch signalling is one of the key pathways in metazoans that regulate cell fate decisions in several systems, including the haematopoietic system. ${ }^{1,2}$ In humans, there are four Notch single-pass transmembrane receptors (Notch1-4) and five transmembrane Notch ligands (Delta-like 1/2/3 and Jagged 1/2). ${ }^{3,4}$ Notch receptors on maturation in the secretary pathway are presented on the cell surface where they interact with their ligands, leading to two successive cleavage of the Notch receptor by a disintegrin and metalloproteinase family metalloproteases followed by $\gamma$-secretase, resulting in the production of Notch1 intracellular domain (NICD), which then enters the nucleus. ${ }^{5}$ In the nucleus, NICD interacts with the DNA-binding protein recombinant signal-binding protein for immunoglobulin- $\kappa \mathrm{J}$ region (RBP- $\mathrm{J} \kappa$; also called CSL). NICD thus displaces the co-repressor from the RBP-J $\kappa$ complex and converts the RBP- $J \kappa$ complex into a transcriptional activator that includes other co activators like mastermind-like protein-1, p300 or p300/CBP-associated factor. Some of the known transcriptional targets of Notch pathway include members of the basic helix-loop-helix Hairy enhancer of split (Hes) factors, Hes-related repressor protein, GATA-3 and Deltex. ${ }^{6}$

In the haematopoietic system, Notch signalling has been known to be important for the proper development of the lymphoid compartment; Notch1, in particular, is known to be crucial for T-cell specification over B-cell lineage, ${ }^{7}$ whereas Notch2 is known to be required for B-cell development. ${ }^{8}$
Moreover, Notch mutations are common in T-cell acute lymphoblastic leukaemia. However, the role of Notch in the myeloid compartment is ambiguous with reports suggesting that Notch1 signalling enhances megakaryocyte differentiation, ${ }^{9-12}$ whereas others indicating the opposite. ${ }^{13-15}$ Notch pathway activates Akt pathway, ${ }^{16,17}$ and reports have also shown the requirement of Akt signalling during megakaryopoiesis. ${ }^{18-22}$ Recently, it has been shown that during murine megakaryopoiesis, Notch signalling elevates Akt phosphorylation. ${ }^{11}$ Whether Notch induces megakaryopoiesis in an Akt-dependent fashion in the context of human haematopoiesis remains to be seen.

Enhancer of zeste homolog-2 (EZH2) is one of the Polycomb group of proteins that functions as a part of chromatin remodelling complex known as Polycomb repressor complex (PRC). EZH2 trimethylates Histones on lysine residues, specifically $\mathrm{H} 3 \mathrm{~K} 27$, resulting in the subsequent repression by chromatin compaction. ${ }^{23} \mathrm{EZH} 2$ has been shown to be essential for B-cell development ${ }^{24}$ and has been found to be upregulated in proliferating mantle cell

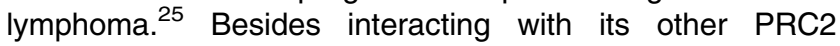
partners, EZH2 localizes in the cytoplasmic compartment of $\mathrm{T}$ cells where it interacts with vav1, thereby regulating T-cell-receptor-mediated signalling. ${ }^{26}$ Interestingly, recent reports have suggested a plausible role for EZH2 in myeloid lineage. ${ }^{27-29}$

Structural Genomics Division, Saha Institute of Nuclear Physics, 1/AF Bidhannagar, Kolkata, West Bengal 700064, India

${ }^{*}$ Corresponding author: S Banerjee, Structural Genomics Division, Saha Institute of Nuclear Physics, 1/AF Bidhannagar, Kolkata, West Bengal 700064, India. Tel: +91 332337 0425; Fax: +91 332337 4637; E-mail: subrata.banerjee@saha.ac.in

Keywords: notch1; Akt; megakaryocyte; EZH2; LIMK1

Abbreviations: NICD, Notch1 intracellular domain; EZH2, enhancer of zeste homolog-2; PDI, protein disulfide isomerase; LIMK1, LIM domain kinase-1; Co-IP, co-immunoprecipitation; RBP-J $\kappa$, recombinant signal-binding protein for immunoglobulin- $\kappa \mathrm{J}$ region; GSI, gamma secretase inhibitor; MALDI, matrix-assisted laser desorption ionization; MS, mass spectrometry; TPA, 12-O-tetradecanoylphorbol 13-acetate; HPRT1, hypoxanthine phosphoribosyl transferase-1

Received 17.2.12; revised 11.7.12; accepted 11.7.12; Edited by H-U Simon 
Our present study shows that Notch1-induced early megakaryopoiesis is Akt-dependent. During this process, the expression of EZH2 was upregulated in the cytoplasmic compartment where it interacts with a novel interactor - LIM domain kinase-1 (LIMK1). This reduced Ser3 phosphorylation of cofilin, thereby increasing cofilin activity and hence lowering filamentous actin content within these cells. Notch1 thus not only mediates early megakaryopoiesis but also prepares these cells for the subsequent morphological changes.

\section{Results}

Notch1-induced early megakaryopoiesis is Akt-dependent. To investigate the effect of activated Notch1 pathway on the megakaryocytic lineage, NICD was transfected into K562 and HEL cells. In both these cell lines, NICD caused an upregulation of two megakaryocyte markers - CD61 and CD42b markers (Figure 1a and Table 1). Gamma secretase inhibitor (GSI) was used to reduce the intracellular NICD levels in HEL and K562 cells, which resulted in reduced CD42b expression (Figure $1 \mathrm{~b}$ and Table 1). As Notch pathway is known to activate the Akt pathway, ${ }^{16}$ we looked into the Akt phosphorylation status. We observed an increase in Akt phosphorylation upon NICD transfection in K562 and HEL cells (Figure 1c). Further, GSI-treated K562 and HEL cells showed a decrease in Akt phosphorylation (Figure 1d). To understand the importance of Akt in megakaryocytic differentiation, a constitutively active form of Akt (myristoylated, myr-Akt) was transfected into K562 and HEL cells. In both these cell lines, myr-Akt caused increased expression of CD61 and CD42b (Figure 1e and Table 1). In addition, inhibition of Akt by the PI3K/Akt inhibitor LY294002 also reduced CD42b levels in K562 cells transfected with NICD (mean fluorescence intensity with respect to $\mathrm{K} 562 \mathrm{NICD}$ is $0.83 \pm 0.08, P<0.001$, $n=3$; Figure 1f). Further, K562 NICD had a CD61 content resembling that of $\mathrm{K} 562$ within the first $2 \mathrm{~h}$ of 12 O-tetradecanoylphorbol 13-acetate (TPA) treatment (Figure $1 \mathrm{~g}$ and Table 2). Thus, NICD-induced early megakaryopoiesis is mediated by the Akt pathway.

Changes in transcription factor brought on by NICD. The expression of some key genes and transcription factors required for erythroid-megakaryocytic differentiation was also analysed in K562 cells transfected with NICD. PU.1, EKLF and myb were downregulated in the presence of NICD, whereas integrin- $\alpha \mathrm{V}$ and integrin- $\beta 3$ was upregulated. Expression of megakaryocyte specifying transcription factors like GATA-1, FOG1, Fli1 and NF-E2 decreased on NICD transfection reminiscent of a similar reduction after TPA treatment (Figure 2a). Further, treatment with TPA for different time points showed an initial decrease in the expression of GATA2 followed by a sustained increase (Figure 2b). As GATA-2 can mediate megakaryopoiesis in the absence of GATA-1, ${ }^{30}$ our results indicate that at least in K562 NICD system, GATA-2 overrides the effect of GATA-1. Our data also shows similarity in the regulation of NICDinduced Hes/Hey genes between K562 cells transfected with NICD and K562 cells treated with TPA (Figure 2c). Together, these data point towards an early megakaryocytic-lineage specification by NICD. To verify the biological relevance of Notch signalling during megakaryopoiesis, CD34 ${ }^{+}$haematopoietic stem and progenitor cells were isolated from human cord blood samples and were cultured in the presence of a cocktail of cytokines, and analysed for the expression of CD61 and CD42b (Figure 2d). The expression of the Notch pathway genes was analysed at day 10 of sorted CD61 ${ }^{+}$ $\mathrm{CD}_{2} \mathrm{~b}^{+}$cells compared with day 0 of megakaryocyte culture. Results show an increase in the mRNA expression of Jag1. Expression of Jag2 remained undetected at day 0 , whereas its expression was observed at day 10 in four independent samples. Expression of megakaryocytic genes showed an increase at day 10 compared with day 0 , whereas transcription of EKLF, an erythroid lineage-specific transcription factor decreased significantly (Figure 2e). Together, the above data not only demonstrates the importance of NICD in mediating megakaryopoiesis, but also shows a correlation between Notch pathway and megakaryopoiesis during in-vitro differentiation of $\mathrm{CD} 4^{+}$cells into megakaryocyte lineage.

NICD increases cytoplasmic EZH2 levels during early megakaryopoiesis. In light of recent reports linking EZH2 to myeloid lineage, we investigated the role of $E Z H 2$ in megakaryopoiesis. NICD-transfected K562 cells were found to have elevated EZH2 levels. Surprisingly, no significant change in histone-3 trimethylated on lysine 27 (H3K27me3) levels was detected (Figure 3a). The nuclear and cytoplasmic distribution of the protein was therefore analysed. Nuclear expression of EZH2 was found to be fairly unchanged, whereas cytoplasmic levels increased with NICD expression (Figure 3b). As NICD increased Akt phosphorylation, we checked whether expression of myr-Akt could mimic this effect of NICD, and found a similar upregulation of EZH2 in the absence of NICD (Figure 3c). Also, blocking Akt phosphorylation in K562 NICD cells with LY294002 caused a decrease in the transcript levels (Supplementary Figure S1A) as well as in the cytoplasmic EZH2 levels (Figure $3 d$ ). The effect of Notch on cytoplasmic EZH2 levels was also verified in Jurkat cells as Notch pathway is activated in these cells and previous report had also indicated that T-cell receptor signalling is affected by cytoplasmic EZH2. ${ }^{26}$ Our data shows that both phosphorylation of Akt and cytoplasmic EZH2 levels decreased upon GSI treatment of Jurkat cells (Supplementary Figure S1B). In K562 cells, EZH2 accumulates in the cytoplasm during early time points of TPA treatment (Figure 3e). To investigate the role of Akt in megakaryopoiesis, phosphorylated Akt levels were checked at different time points of TPA treatment of K562 cells. Results show an increase in phosphorylated Akt during early time points of differentiation, indicating that upregulation of EZH2 is brought about by NICD-induced Akt phosphorylation. A similar expression of EZH2 was seen with HEL cells treated with TPA for different time points and HEL cells transfected with NICD (Figure 3f). Thus, early megakaryocyte specification by NICD mediated by phosphorylated Akt involves the upregulation of $\mathrm{EZH} 2$, which accumulates more in the cytoplasm of these cells. EZH2 levels were also checked in in-vitro megakaryocyte cultures at day 5 (CD61 ${ }^{+}$ $\left.\mathrm{CD}_{2} \mathrm{~b}^{-}\right)$and day $10\left(\mathrm{CD} 61^{+} \mathrm{CD}^{-} \mathrm{b}^{+}\right)$of sorted cells. 

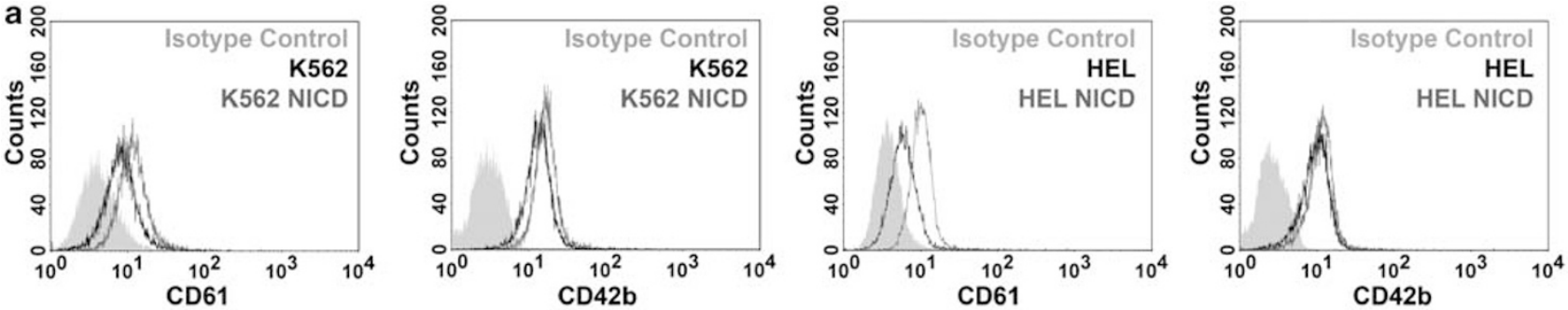
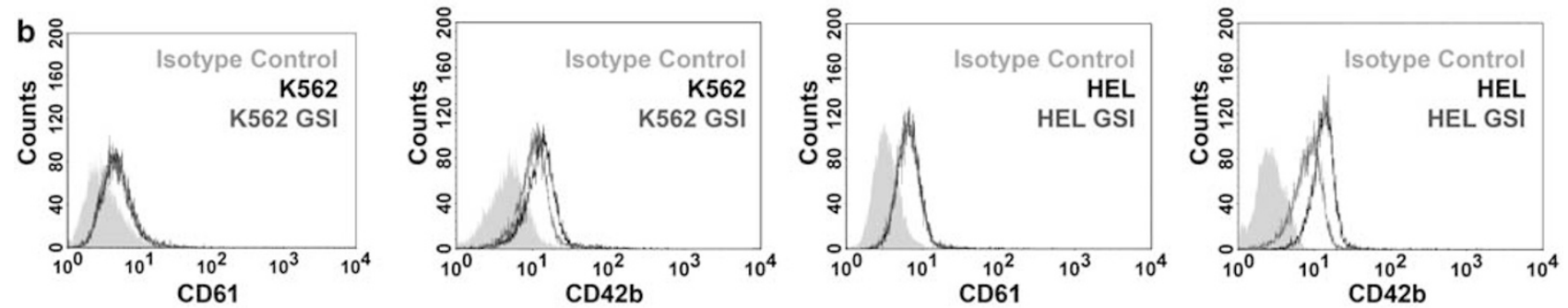

C
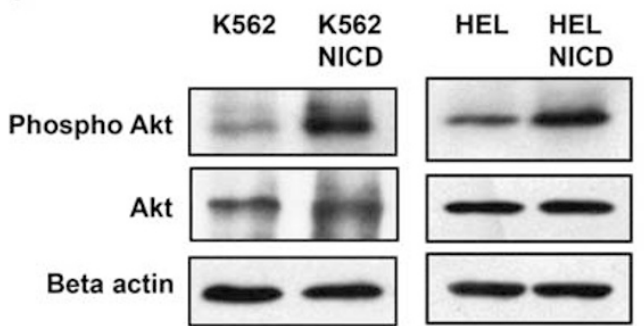

d

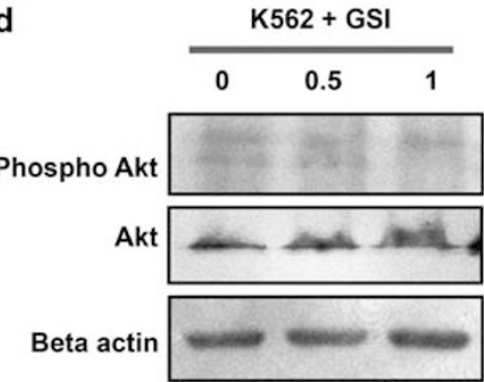

HEL HEL

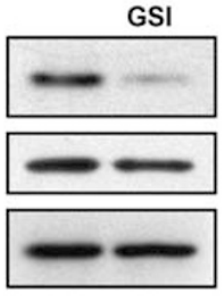

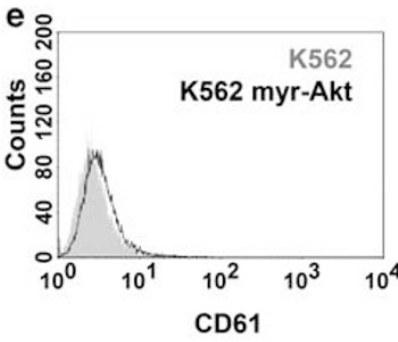

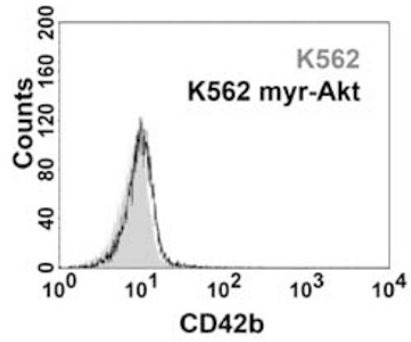

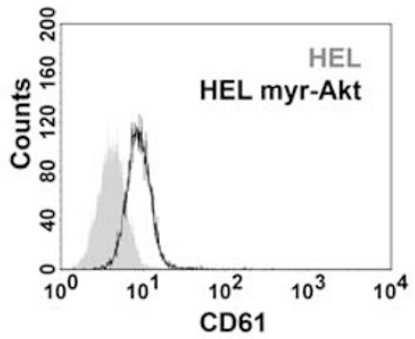
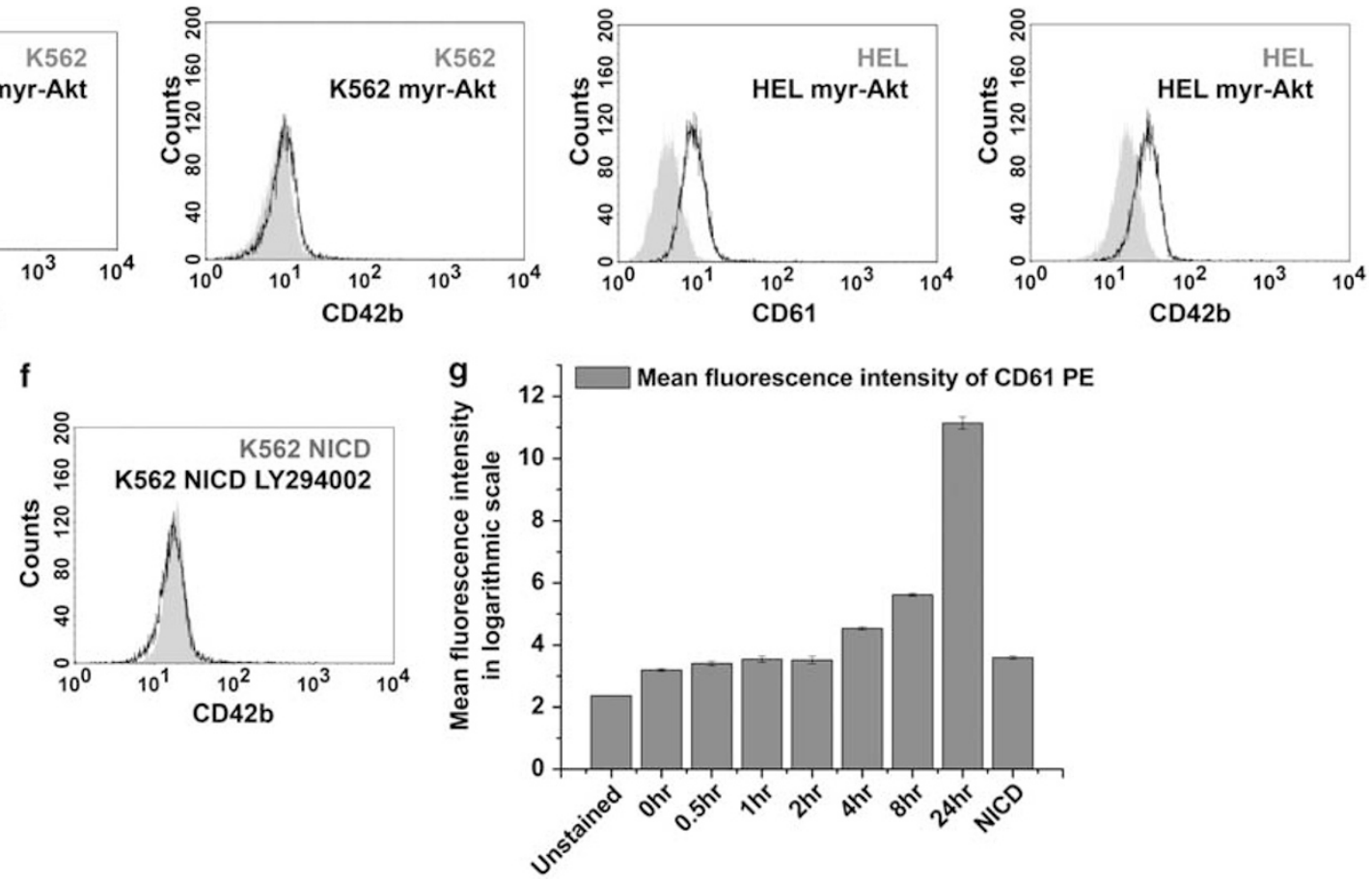

Figure 1 NICD induces early megakaryocytic differentiation. (a) Flow cytometric analysis of CD61 and CD42b markers in K562, K562 NICD, HEL and HEL NICD. (b) Flow cytometric analysis of CD61 and CD42b markers in K562, HEL with/without GSI treatment. (c) Western blot of whole-cell lysates of K562, K562 NICD, HEL and HEL NICD showing the relative expression of phospho-Akt and Akt. (d) Phospho-Akt and Akt expression in GSI-treated K562 and HEL cells. For K562 cells, 0.5 and $1 \mu$ M GSI was used, whereas for HEL cells, $1 \mu \mathrm{M}$ GSI was used. Densitometric analysis of the blots is provided in the Supplementary Information S3. (e) Flow cytometric analysis of CD61 and CD42b in K562, K562 myr-Akt, HEL and HEL myr-Akt. (f) Flow cytometric analysis of CD42b-stained K562 NICD cells and it's LY294002 (20 $\mu$ M)-treated counterpart. (g) Mean fluorescence intensity of CD61-stained K562, K562 NICD and K562 treated with TPA for different time points 
Table 1 Megakaryocyte-specific marker expression

\begin{tabular}{|c|c|c|c|c|}
\hline \multirow[b]{2}{*}{ Treatment/transfection } & \multicolumn{2}{|c|}{ K562 } & \multicolumn{2}{|c|}{ HEL } \\
\hline & CD61 & CD42b & CD61 & CD42b \\
\hline $\begin{array}{l}\text { NICD } \\
\text { GSI } \\
\text { myr-Akt }\end{array}$ & $\begin{array}{c}1.3 \pm 0.03^{\star} \\
0.97 \pm 0.086^{\star \star \star} \\
1.14 \pm 0.012^{\star \star}\end{array}$ & $\begin{array}{c}1.23 \pm 0.02^{\star} \\
0.8 \pm 0.047^{\star \star} \\
1.17 \pm 0.03^{\star}\end{array}$ & $\begin{array}{l}1.55 \pm 0.016^{\star} \\
1.09 \pm 0.016^{\star \star} \\
1.57 \pm 0.08^{*}\end{array}$ & $\begin{array}{l}1.15 \pm 0.015^{\star \star} \\
0.82 \pm 0.03^{\star \star} \\
1.62 \pm 0.09^{\star \star}\end{array}$ \\
\hline
\end{tabular}

K562 and HEL cells were transfected with NICD and myr-Akt, and fold increase in the expression of CD61 and CD42b was analysed. K562 and HEL cells were subjected to $1 \mu \mathrm{M}$ GSI treatment and the megakaryocyte marker expression was analysed. Expression of CD61 and CD42b in K562 was taken as 1 and the fold changes of treated/transfected cells were calculated. Mean \pm s.e.m. of three independent experiments are shown ( $\left.{ }^{\star} P<0.0001,{ }^{\star \star} P<0.001,{ }^{\star \star \star} P<0.01\right)$.

Table 2 CD61 expression in TPA-treated K562 cells

\begin{tabular}{lc}
$\begin{array}{l}\text { K562 }+32 \text { nM TPA } \\
\text { (hours of treatment) }\end{array}$ & $\begin{array}{c}\text { Fold change in mean fluorescence } \\
\text { intensity with respect to K562 }\end{array}$ \\
\hline 0.5 & $1.12 \pm 0.015^{\star}$ \\
1 & $1.23 \pm 0.014^{\star}$ \\
2 & $1.2 \pm 0.01^{*}$ \\
4 & $1.43 \pm 0.018^{\star \star}$ \\
8 & $1.86 \pm 0.047^{\star \star}$ \\
24 & $3.59 \pm 0.086^{\star \star}$
\end{tabular}

K562 cells were treated with TPA for different time points and fold change in the expression of CD61 was analysed. Expression of CD61 in K562 was taken as 1 and fold changes were calculated with respect to K562. Mean \pm s.e.m. of three independent experiments are shown $\left({ }^{\star} P<0.005\right.$, $\left.{ }^{\star \star} P<0.001\right)$.

The transcript levels of EZH2 were found to be higher compared with day 0 of cultures (Figure $3 \mathrm{~g}$ ). Also, EZH2 content measured in the bone marrow mononuclear cells stained for two megakaryocytic markers CD61 and CD42b showed that $\mathrm{CD} 61^{+} \mathrm{CD} 42 \mathrm{~b}^{-}$population had lower $\mathrm{EZH} 2$ content than $\mathrm{CD} 61{ }^{+} \mathrm{CD} 42 \mathrm{~b}{ }^{+}$population (Figure $3 \mathrm{~h}$ ), again indicating the requirement of $\mathrm{EZH} 2$ during megakaryopoiesis. Thus, we have for the first time illustrated the importance of NICD in upregulation of cytoplasmic EZH2. We have also shown that this effect is seen during early stages of megakaryocyte differentiation.

Novel cytoplasmic partners of EZH2. EZH2 is known to form a functional methyl transferase complex with Eed and Suz12 in the cytoplasm. The only known non-PRC protein that interacts with $\mathrm{EZH} 2$ in the cytoplasm is vav1. ${ }^{31}$ The expression of vav1 was found to decrease after NICD transfection (Figure 4a). To elucidate the function of EZH2, we attempted to find the other cytoplasmic partners of EZH2 by co-immunoprecipitation (Co-IP) of EZH2 followed by matrix-assisted laser desorption ionization (MALDI) MS/MS-based identification of the immunoprecipitates. Five proteins were thus identified - PDIA1, LIMK1, heat-shock protein $90 \beta$, tubulin- $\alpha 6$ variant and gamma actin. Details of the MALDI MS/MS analysis are given in the Supplementary Information S2. The interaction of cytoplasmic EZH2 with LIMK1 and protein disulphide isomerase (PDI) was confirmed by Co-IP experiment in which antibody against LIMK1/PDI was used to pull down EZH2 (Figure 4b). The interactions were further confirmed by reverse immunoprecipitation (Supplementary Figure S1C and D). In addition, immunocytochemisty confirmed that EZH2 and LIMK1 colocalized in the cytoplasm of NICD-transfected K562 cells
(Figure 4c). Thus, two new cytoplasmic interactors of EZH2 were identified - LIMK1 and PDIA1.

Cytoplasmic EZH2 induces changes in filamentous actin content. To understand the function of EZH2 in the cytoplasmic compartment of these cells, the interactors were further probed. In K562 cells transfected with NICD, which show an early megakaryocytic propensity, PDIA1 itself was upregulated. LIMK1 levels however remain unchanged (Supplementary Figure S1E). As LIMK1 and vav1 are both involved in the regulation of actin dynamics, we looked into how NICD affects the other factors in this process. The Rho and Rac GTPases showed altered levels in the presence of NICD, whereas cdc42 levels remain unchanged (Figure 5a). LIMK1 is known to function immediately upstream of the actin polymer-severing protein cofilin. It phosphorylates cofilin, thereby rendering it inactive. ${ }^{32}$ Phosphorylation of cofilin decreased upon NICD activation (Figure 5b). Further, total filamentous actin content decreased marginally in NICDtransfected cells (mean fluorescence intensity with respect to $\mathrm{K} 562$ is $0.84 \pm 0.015, n=3$; Figure $5 \mathrm{c}$ ). To address the question whether $\mathrm{EZH} 2$ has any role in inducing changes in the actin cytoskeleton structure, EZH $2 \Delta$ NLS was transfected into K562 cells. This caused a decrease in the phosphorylated cofilin levels (Figure 5d). Also, siRNA against EZH2 showed an increase in phosphorylated cofilin levels (Figure 5e). Together, these results indicate a role for EZH2 in modulating the filamentous actin content within these cells through regulation of cofilin phosphorylation. As Akt pathway mediated the cytoplasmic localization of EZH2, LY294002 was used to inhibit Akt pathway to see its effect on actinfilament-severing proteins. We found that phosphorylated cofilin levels increased upon treatment with LY294002 (Figure 5f). Similar effects were seen with F-actin content in K562 transfected with NICD and K562 NICD-transfected cells treated with LY294002, wherein a decrease in F-actin content brought about by NICD could be reversed with LY294002 treatment (mean fluorescence intensity compared with K562 NICD 1.16 $\pm 0.015, n=3$; Figure 5g). Again, myrAkt caused decreased phosphorylation of cofilin (Figure $5 \mathrm{~h}$ ). Thus, NICD-mediated increase in cytoplasmic EZH2 levels results in decreased filamentous actin content within early megakaryocytes.

\section{Discussion}

The role of Notch pathway during megakaryocyte differentiation has been greatly studied, but there are differences in 
a

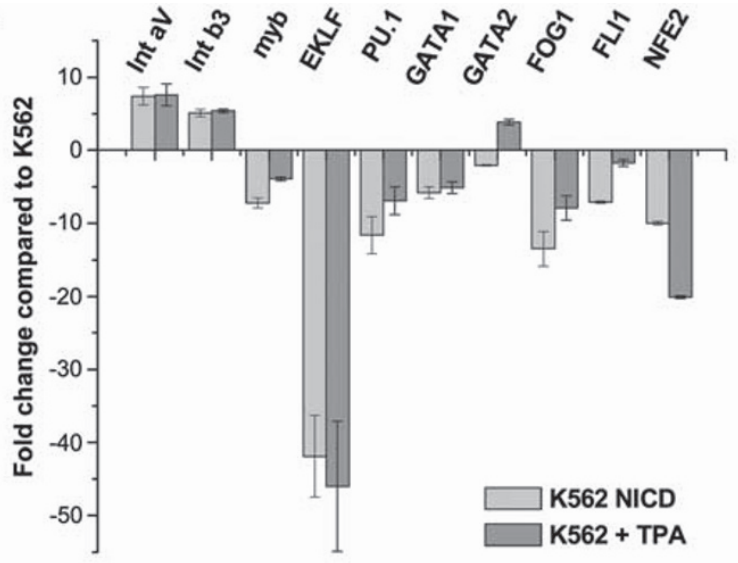

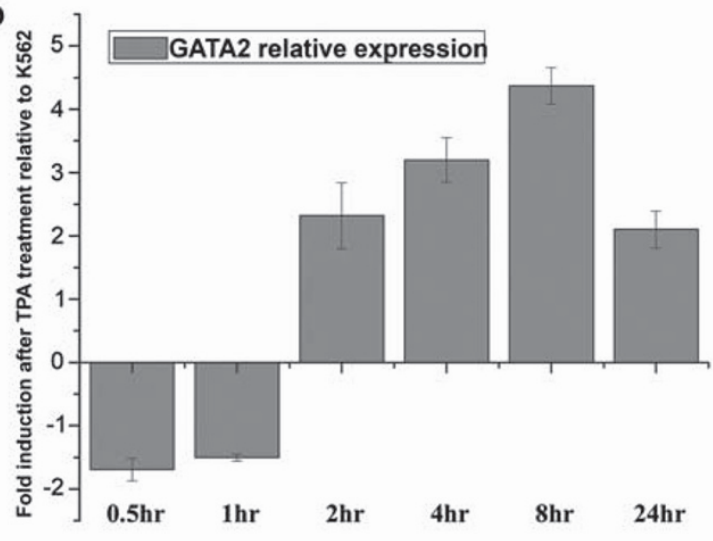

d

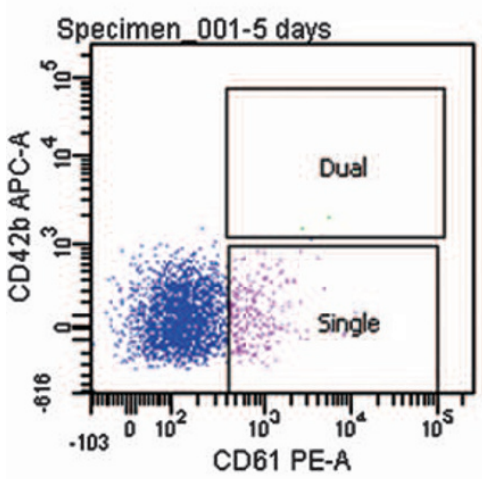

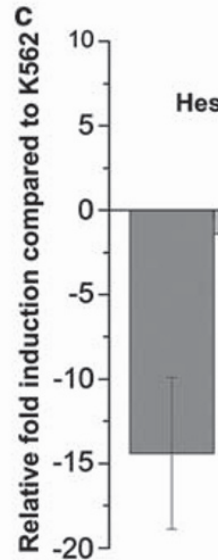

K562 transfected with NICD

K562 treated with TPA

Hes5 Hey1

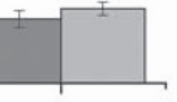

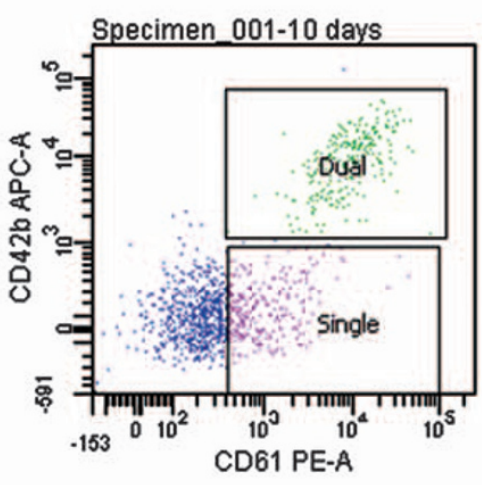

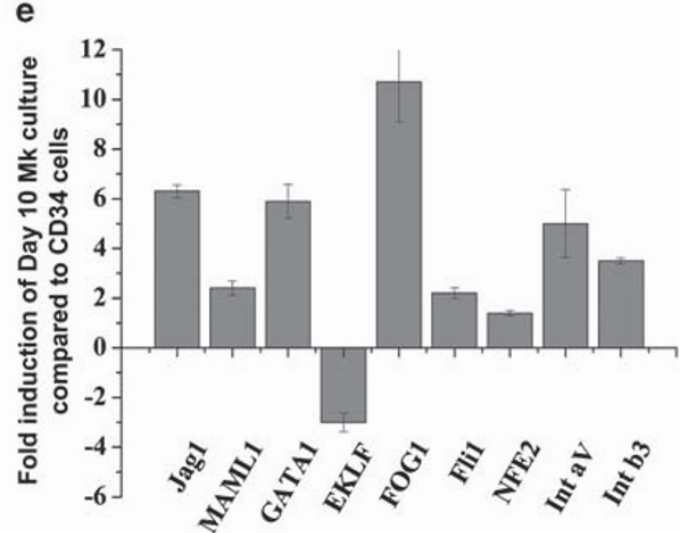

Figure 2 NICD induced change in transcription factors. (a) Relative gene expression in K562 transfected with NICD and K562 treated with TPA compared with K562 cells. The mRNA expression of each gene was normalized to the expression of HPRT1 mRNA. Data represents the mean \pm s.e.m of four independent experiments $(P<0.001$, $n=4)$. (b) Relative gene expression of GATA-2 at different time points of TPA treatment as seen by qRT-PCR. Data represents the mean \pm s.e.m of three independent experiments $(P<0.002, n=3)$. (c) Relative gene expression where data represents the mean \pm s.e.m of three independent experiments $(P<0.002, n=3)$. (d) $C D 34^{+}$cells isolated from cord blood induced into megakaryocytic lineage. $\mathrm{CD} 61^{+} \mathrm{CD} 42 \mathrm{~b}^{-}$and $\mathrm{CD} 61^{+} \mathrm{CD} 42 \mathrm{~b}^{+}$populations were sorted at the end of 5 days and 10 days of megakaryocytic culture. (e) Differentiation was assessed with surface markers CD61 and CD42b. qRT-PCR levels of Notch1 pathway genes and megakaryocyte-specific transcription factors at day $10\left(\mathrm{CD} 61^{+} \mathrm{CD}_{22} \mathrm{~b}^{-}\right)$megakaryocyte cultures compared with day 0 cultures. Data represents mean \pm s.e.m of three independent experiments $(P<0.005)$

terms of the results, with reports suggesting that Notch pathway enhances megakaryopoiesis, ${ }^{9-12}$ whereas others suggesting the reverse. ${ }^{13-15}$ Despite these differences, the reports clearly indicate that Notch pathway is a crucial determinant during megakaryopoiesis in both human and murine systems. In this study, we have addressed the question of Notch-induced megakaryopoiesis and its impact on the cellular actin network.

As observed by the surface marker expression of CD61 and CD42b, NICD was found to induce megakaryocytic differentiation of $\mathrm{K} 562$ and HEL cells. In addition, during in-vitro megakaryocyte differentiation from cord blood $\mathrm{CD}_{3}{ }^{+}$cells, 

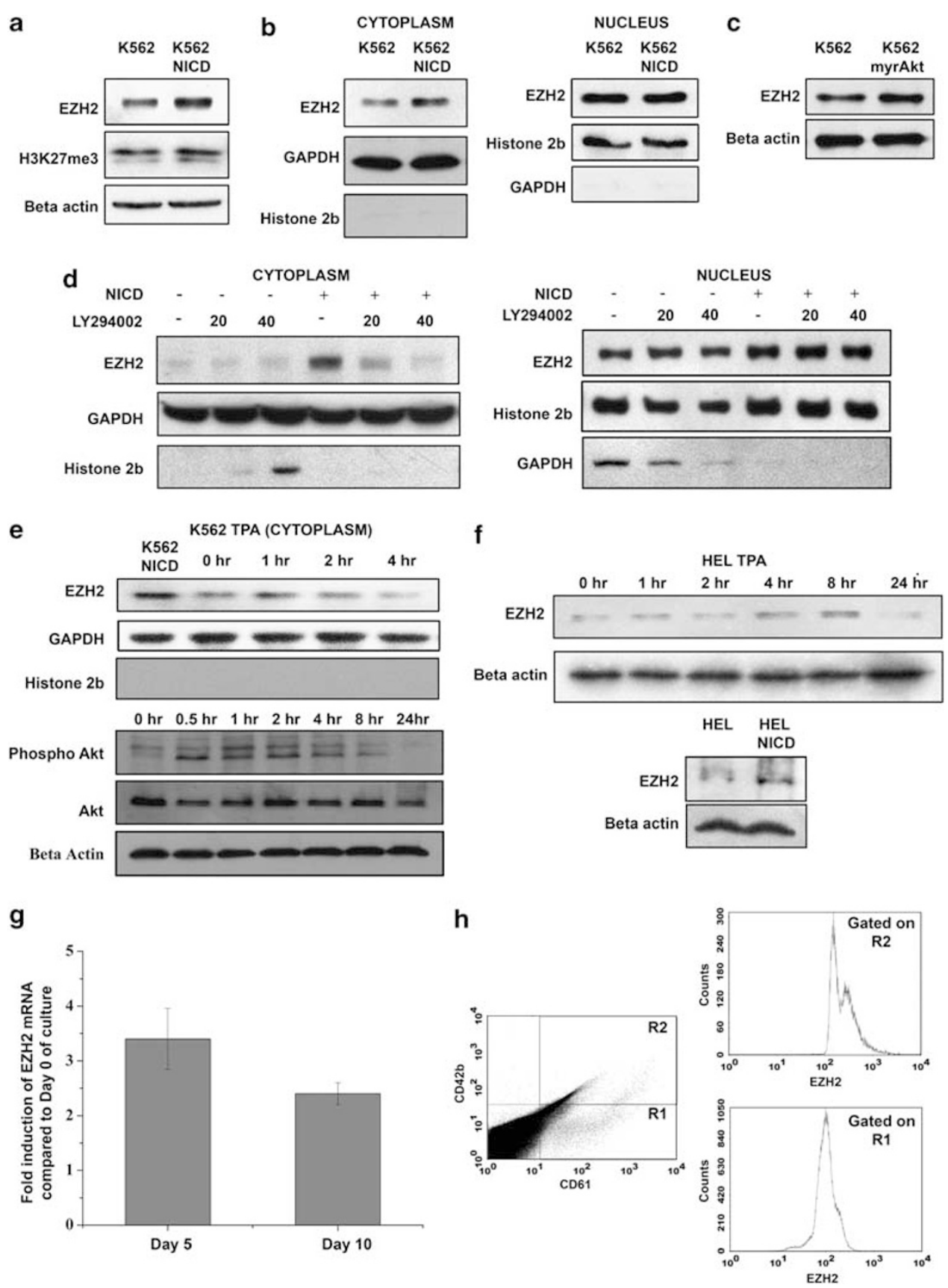

Figure 3 NICD induces Akt-dependent upregulation of cytoplasmic EZH2. (a) Western blot of whole-cell lysate of K562 and K562 NICD showing the expression of EZH2 and H3K27me3 (histone-3 trimethylated on lysine 27). (b) EZH2 expression in the nuclear and cytoplasmic fractions of K562 and K562 NICD. (c) Western blot showing the expression of EZH2 in K562 and K562 myr-Akt. (d) EZH2 expression in the nuclear and cytoplasmic fractions of K562 cells treated with the mentioned concentrations of LY294002 with/without NICD transfection. (e) EZH2 expression in the cytoplasmic compartments of K562 NICD and K562 at different time points of TPA treatment. Lower panel shows phosphorylation levels of Akt at different time points of TPA treatment. (f) EZH2 expression in the whole-cell lysates of HEL at different time points of TPA treatment. Lower panel shows the expression of EZH2 in NICD-trasfected HEL cells. Densitometric analysis of the blots is provided in the Supplementary Information S3. (g) qRT-PCR of EZH2 levels in day 5 and day 10 of megakaryocyte cultures compared with day 0 cultures. Data represents mean \pm s.e.m of three independent experiments $(P<0.001)$. (h) Mononuclear cells isolated from bone marrow aspirates and stained with $\mathrm{CD} 61$ and $\mathrm{CD} 42 \mathrm{~b}$ to identify megakaryocyte population and anti-EZH2 antibody. The level of EZH2 was determined from $\mathrm{CD} 61{ }^{+} \mathrm{CD}_{2} 2 \mathrm{~b}^{-}$(R1)- and $\mathrm{CD} 61{ }^{+} \mathrm{CD} 42 \mathrm{~b}^{+}$(R2)-gated cells

Notch1 pathway genes such as Jag1 and Jag2 were upregulated in conjunction with megakaryocyte specifying transcription factors. It is known that GATA-1, Fog1, Fli1 and NF-E2 are required for megakaryocytic differentiation. GATA1 is dispensable for the onset of megakaryopoiesis, but is required for the maturation of megakaryocytes. ${ }^{33,34}$ Similarly, mice lacking p45NF-E2 produced megakaryocytes that showed defective platelet production, although other parameters of megakaryocyte maturation like endomitosis and expression of GPIlb occurred. ${ }^{35}$ Also, loss of Fli1 in adult mice 

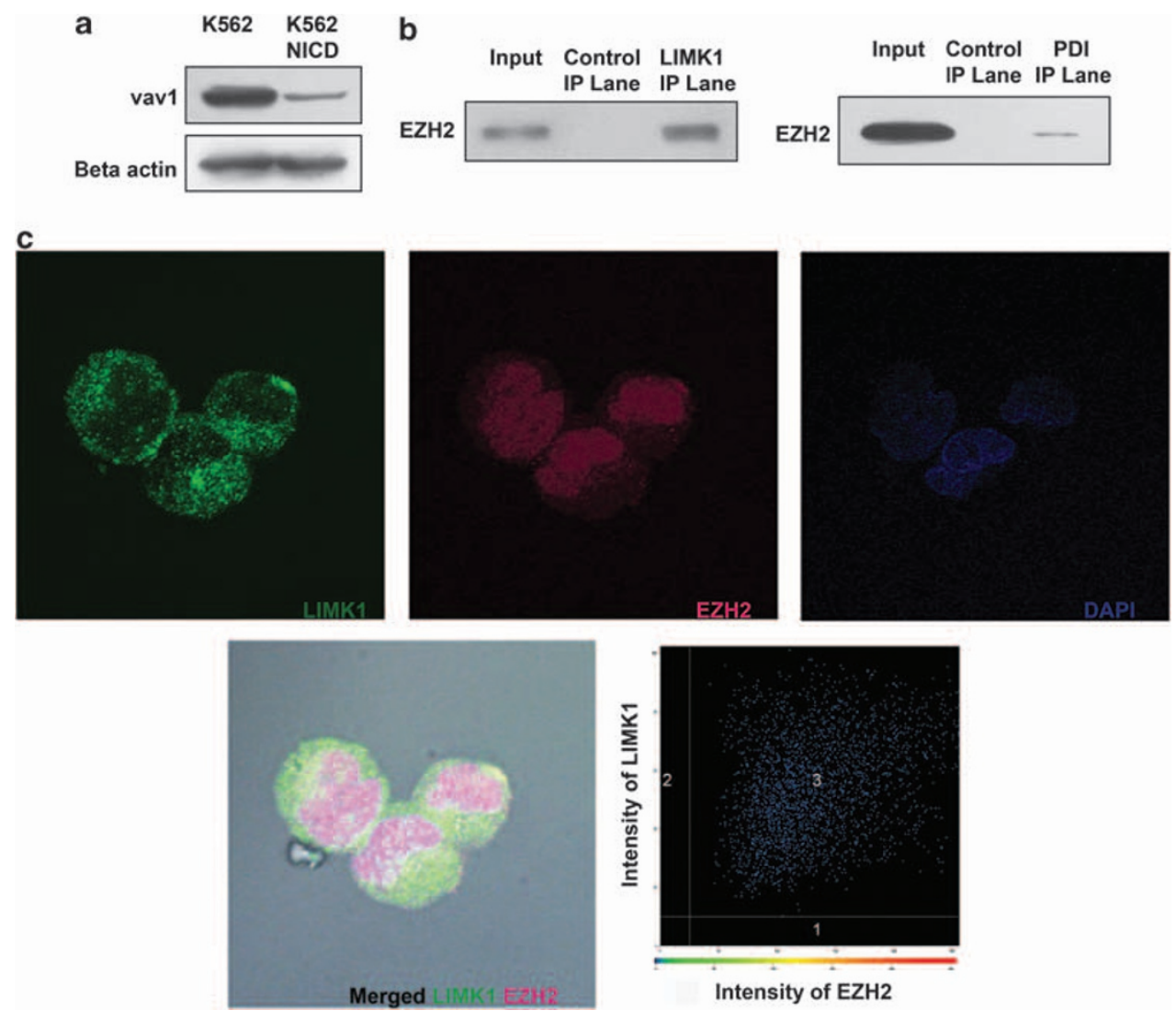

Figure 4 Cytoplasmic interactors of EZH2. (a) Western blot of whole-cell lysates showing the expression of vav1 in NICD-transfected K562 cells. Densitometric analysis of the blots is provided in the Supplementary Information S3. (b) Co-IP results in which antibody against LIMK1 was used to pull down LIMK1-protein complex and probed for EZH2, and antibody against PDI was used to pull down PDI-protein complex and probed for EZH2. (c) Immunocytochemistry of K562 NICD cells. Confocal images of LIMK1-, EZH2- and DAPI-stained cells were analysed for colocalization of LIMK1 and EZH2. $(R=0.57$, s.e.m. $\pm 0.047, P$-value $<0.001)$

resulted in mild thrombocytopenia associated with a marked reduction of megakaryocytic progenitors and maturation defect of megakaryocytes present in bone marrow. ${ }^{36}$ Thus, although these transcription factors are essential for proper megakaryocyte development, their absence does not lead to a complete ablation of megakaryocytes. The decrease in the mRNA levels of these transcription factors in NICD-induced $\mathrm{K} 562$ cells as well as TPA-treated $\mathrm{K} 562$ cells indicates that these proteins are already present in K562 cells in amounts sufficient to induce changes necessary for megakaryopoiesis (GATA-1 protein levels in NICD-transfected K562 cells shown in Supplementary Figure S1G). Further, the mRNA levels of these transcription factors increases during in-vitro megakaryocyte production from cultured $\mathrm{CD} 34^{+}$cells as shown in Figure 2e. Interestingly, loss of GATA-1, FOG1 and NF-E2 has also been shown to affect erythropoiesis. ${ }^{37-39} \mathrm{~A}$ bipotent cell line such as K562, which can differentiate either towards erythroid or megakaryocyte lineage, expresses all these transcription factors as shown by qRT-PCR data (Figure 2a). In such cells, downregulation of these transcription factors may be helpful in induction of megakaryopoiesis in preference to erythropoiesis. It is known that GATA-2 can mediate megakaryopoiesis in the absence of GATA-1. ${ }^{35}$ Our data shows that GATA-2 expression was upregulated in TPA-treated K562 cells. Further, treatment with TPA for different time points showed an initial decrease in the expression of GATA-2 followed by a sustained increase. These results are in agreement with previous reports ${ }^{36,37}$ in which overexpression of GATA-2 promoted megakaryocytic differentiation at the expense of erythroid differentiation. Also, integrin- $\alpha \mathrm{V}$ and integrin- $\beta 3$ transcript level was upregulated. Interestingly, integrin- $\beta 3$ is also a known surface marker of megakaryocytes and platelet, also called CD61, and has been widely used to detect early maturation stages of megakaryocytes and in this study integrin- $\beta 3$ expression level was used as a marker for characterizing megakaryocytic differentiation. Thus, the CD61 levels and megakaryocyte-specific transcription factor levels point towards an early megakaryocyte specification by NICD. This process was found to be Akt-dependent. In fact, dependence of megakaryopoiesis on Akt was previously demonstrated in murine megakaryopoiesis. ${ }^{11}$ Our data indicates that Akt is crucial for Notch-dependent megakaryopoiesis, as inhibition of Akt in NICD-transduced cells leads to lower megakaryocyte-specific markers CD61 and CD42b.

The early indications that EZH2 could be involved in myeloid lineage specification came from the genomic location of EZH2 within the 7q35 locus also known for its involvement in malignant myeloid disorders. ${ }^{40}$ Later reports had indicated a possible role for $\mathrm{EZH} 2$ in megakaryocyte development and 


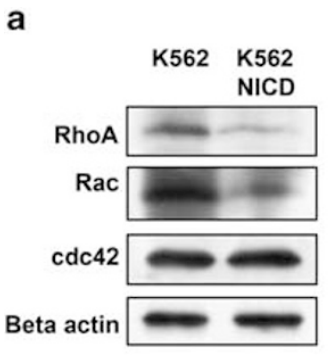

b

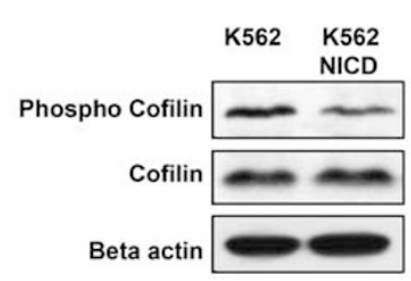

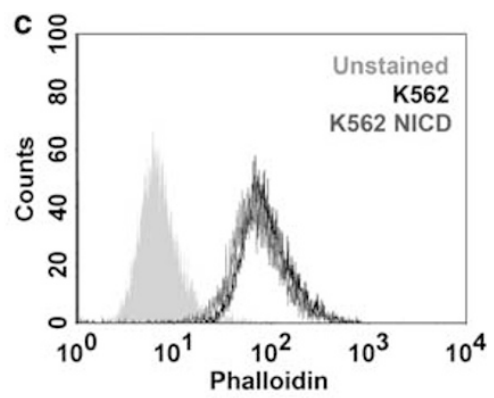

xate

d

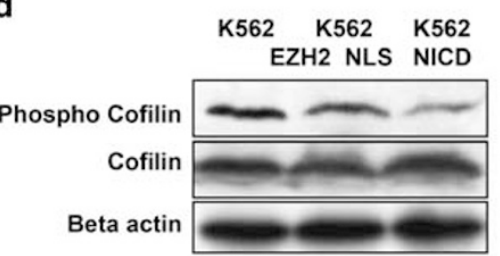

e

hospho Cofilin

Cofilin

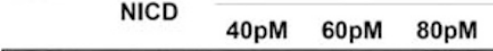

Beta actin
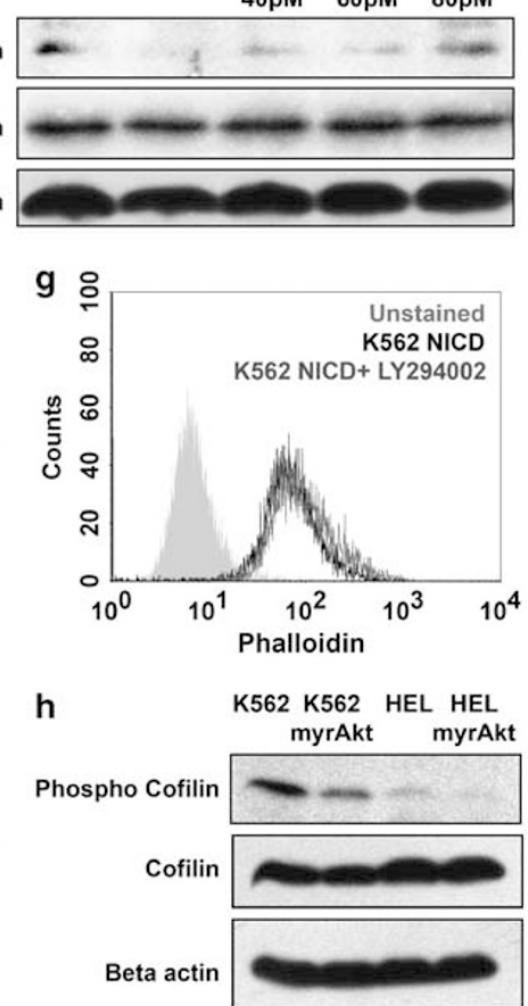

Figure 5 Cytoplasmic EZH2 induced changes in filamentous actin content. (a) Western blots of whole-cell lysates of K562 and K562 NICD representing changes in Rho/Rac/cdc42 GTPases. (b) Western blots of whole-cell lysates of K562 and K562 NICD showing the changes in expression of Ser3 phosphorylated cofilin. (c) Filamentous actin content in K562 and K562 NICD cells. (d) Western blot of whole-cell lysates of K562, K562 EZH2 $\mathrm{NLS}$ and K562 NICD analysed for the expression of Ser3phosphorylated cofilin and cofilin. (e) Western blot of whole-cell lysates of K562, K562 NICD and K562 NICD treated with different concentrations of EZH2 siRNA was analysed for the expression of Ser3-phosphorylated cofilin and cofilin. (f) Western blots of whole-cells lysates of K562 cells treated with LY294002 with the mentioned concentrations in $\mu \mathrm{M}$ with/without NICD transfection. (g) Filamentous actin content in K562 NICD and K562 NICD treated with LY294002. (h) Western blots of whole-cell lysates of K562, K562 myr-Akt, HEL and HEL myr-Akt representing changes in phosphorylation of cofilin. Densitometric analysis of the blots is provided in the Supplementary Information S3

maturation. In particular, the expression of EZH2 was found to increase in the newly produced megakaryocytic lineage cells after conditional eradication of $\mathrm{CD} 41^{+}$cells. $^{27}$ Further, patients with myelodysplastic syndrome, who usually express a dysplasia of erythroid/megakaryocytic compartment, were found to harbour somatic mutations of $E Z H 2$ gene. ${ }^{28} \mathrm{~A}$ recent report also points out the importance of $E Z H 2$, wherein conditional deletion of EZH2 in mice fetal livers reduced the numbers of myeloid progenitors, including megakaryocyte erythocyte progenitors. ${ }^{29}$ We thus investigated the role of $\mathrm{EZH} 2$ in megakaryopoiesis. Our results show for the first time that $\mathrm{EZH} 2$ is upregulated during the early megakaryocytic differentiation event and this is accomplished by the Notch1 pathway in an Akt-dependent manner. $\mathrm{EZH} 2$ is upregulated in the NICD-transfected K562 and HEL cells. Moreover, EZH2 expression increases during early time points of TPA-induced differentiation of both K562 and HEL cells. This correlates with the levels of phosphorylated Akt that also shows similar increase during TPA-induced differentiation. EZH2 was previously shown to localize in the cytoplasmic compartment of cells. ${ }^{26}$ Here we show that EZH2 is found in the cytoplasm of $\mathrm{K} 562$ cells and it is this cytoplasmic $\mathrm{EZH} 2$, which increases during the first hour of TPA-induced K562 differentiation. Moreover, cytoplasmic EZH2 levels increased upon NICD 
transfection of K562 cells, whereas the nuclear levels of the protein remained unchanged, a result well corroborated by the level of trimethylation of histone3 (H3K27me3). These data not only point towards an early event of megakaryocytic differentiation brought on by NICD, but also highlight the importance of Akt signalling in increasing the cytoplasmic $\mathrm{EZH} 2$ levels. Further, during in-vitro megakaryopoiesis from $\mathrm{CD} 4^{+}$cells, EZH2 transcript levels were found to increase. Such an increase was also seen when bone marrow cells were analysed for the expression of CD61 and CD42b, and then compared for the expression of EZH2.

$\mathrm{EZH} 2$ is known to form a functional complex with its PRC2 partners Eed and Suz12 in the cytoplasm. Previous reports had shown that EZH2 could interact with vav1 and modulate T-cell receptor signalling. ${ }^{26}$ In this study, we tried to determine the other cytoplasmic interactors of EZH2. PDIA1 and LIMK1 emerged as the two new protein interactors. PDI, a known endoplasmic reticulum marker, functions as a protein disulphide isomerase, and hence, it derives its name. It has long been known to localize in the cytoplasm of megakaryocytes. In platelets, $\mathrm{PDI}$ is primarily found on the plasma membrane. ${ }^{41}$ Also, PDI is known to facilitate platelet aggregation. ${ }^{42}$ In our system, PDI was found to be upregulated in NICD-transfected K562 cells, which themselves acquire a megakaryocyte specification, and thus, an increase in PDI content in Notchtransfected K562 cells only further points to the role of Notch in facilitating megakaryocyte lineage. LIMK1 is known to function immediate upstream of the actin polymer-severing protein cofilin. It phosphorylates cofilin, thereby rendering it inactive. Here we propose a mechanism by which NICD increases the cytoplasmic levels of $\mathrm{EZH} 2$, which then interacts with LIMK1 resulting in reduced Ser3 phosphorylation of cofilin, which prepares these cells for subsequent changes in cell shape and size necessary for megakaryocyte formation. Together with the ability to increase the cytoplasmic EZH2 levels, NICD also causes changes in the levels of Rho/Rac GTPases that have a crucial role in cell morphology. Of particular interest is the nature of regulation of these small Rho GTPases - RhoA and Rac levels declined, whereas cdc42 levels remained unchanged with NICD induction. Although previous reports have implicated $\mathrm{EZH} 2$ in prostate cancer metastasis, ${ }^{43}$ this is the first attempt at understanding the molecular factors involved in EZH2-mediated remodelling of actin cytoskeleton. This study revealed that NICD-induced early megakaryocytic differentiation was accompanied by an increase in the cytoplasmic levels of EZH2, which resulted in increased cofilin activity and hence altered filamentous actin levels within these cells. Although we have produced evidence for an Akt-dependent increase in cytoplasmic $\mathrm{EZH} 2$ levels, it would however be interesting to investigate how activation of the Akt signalling leads to increased cytoplasmic EZH2 levels. Although, Akt is known to phosphorylate $\mathrm{EZH} 2$ on serine $21,{ }^{44}$ it remains to be seen whether the cytoplasmic localization of the protein is directly controlled by Akt. In conclusion, this study shows that Notch1 pathway leads to Akt-dependent early megakaryopoiesis and is accompanied by increased cytoplasmic EZH2 levels. Finally, we show how cytoplasmic EZH2 changes the filamentous actin content within early megakaryocytes by interacting with LIMK1.

\section{Materials and Methods}

Culture of CD34 ${ }^{+}$cells and cell lines. K562 and HEL cells were cultured in RPMl1640 (Gibco, Carlsbad, CA, USA) with 10\% FBS (Gibco). For isolation of $\mathrm{CD} 34^{+}$cells, mononuclear cells were isolated from cord blood using Percoll (GE Amersham, Buckinghamshire, UK) and CD34 ${ }^{+}$cells were enriched by magnetic separation (Miltenyi Biotech, Bergisch Gladbach, Germany). Purity of the isolated cells was determined using anti-CD34 antibody (BD Biosciences, San Jose, CA, USA). Megakaryocyte culture was initiated using freshly isolated CD34 ${ }^{+}$cells using the protocol. ${ }^{45}$ Megakaryocyte population was sorted at day 5 $\left(\mathrm{CD} 61^{+} \mathrm{CD} 42 \mathrm{~b}^{-}\right)$and day $10\left(\mathrm{CD} 61^{+} \mathrm{CD}_{22} \mathrm{~b}^{+}\right)$of in-vitro culture in a FACS Ariall system (BD Biosciences). All clinical samples were collected under consent, following strict institutional ethical guidelines.

Plasmids constructs. The expression vector for the activated form of Notch1 pCDNA-Myc-HisN1IC (NICD) was gifted by $\mathrm{J}$ Aster (Harvard Medical School). $\mathrm{CMV}(\mathrm{myc}) 3 \mathrm{EZH} 2$ was a kind gift from $\mathrm{T}$ Jenuwein (Max Planck Institute of Immunology and Epigenetics). EZH2 $\triangle$ NLS was subcloned into RFP vector using the primers $5^{\prime}$-GATTAGTCGACGAATGGAAACAGCGAAGGA-3' ${ }^{\prime}$ and 5'-GGTCTGGATCCAAGGCAGCTGTTTCAGAGGA-3'.

RNA extraction and real-time PCR. RNA was isolated using Trizol (Roche, Mannheim, Gemany). The expression level of genes was assessed by quantitative RT-PCR using SYBR Green core PCR reagents (Applied Biosystems, Carlsbad, CA, USA). Hypoxanthine phosphoribosyl transferase-1 (HPRT1) was used to normalize the expression of the genes. The reactions were carried out in 7500 Sequence Detection Systems (Applied Biosystems). Details of the primer sequence are given in the Supplementary Methods.

Reagents and antibodies. Antibodies against EZH2, LIMK1, PDI, vav1, Rac1/2/3, cdc42, cofilin, phospho-cofilin, Akt and phospho Akt were purchased from Cell Signaling Technology (Danvers, MA, USA). Antibody against RhoA was purchased from Abcam (Cambridge, MA, USA). PE-conjugated CD235a was obtained from Serotec (Kidlington, UK), whereas APC-conjugated CD42b, PEconjugated CD61 and PE-conjugated CD34 was purchased from BD Biosciences. Flow cytometric analysis was performed on a BD FACS Calibur platform. EZH2 siRNA was obtained from Sigma (St. Louis, MO, USA) using the sequence specified in Chen et al. ${ }^{46}$ TPA (Sigma) was used at $32 \mathrm{nM}$ concentration for $24 / 48 \mathrm{~h}$.

Co-IP and western blotting. Nuclear and cytoplasmic extracts were prepared using NE-PER Nuclear and Cytoplasmic extraction reagents Pierce (Rockford, IL, USA). Four hundred microgram of K562 NICD-transfected cytoplasmic extract was incubated with $2 \mu \mathrm{l}$ of EZH2 antibody at $4{ }^{\circ} \mathrm{C}$ for $2 \mathrm{~h}$. Twenty microlitre of protein A/G agarose bead (Santa Cruz, Santa Cruz, CA, USA) was added and incubated overnight at $4{ }^{\circ} \mathrm{C}$. The immunoprecipitate was obtained by centrifugation at 5000 r.p.m. for $5 \mathrm{~min}$. The immunoprecipitate was washed thrice and the remaining immunoprecipitate was resolved on $12 \%$ SDS-PAGE. For protein identification, the gel was stained with Blue Silver stain, and the bands obtained were cut for protein identification. For western blotting, cells were harvested and lysed in cold RIPA buffer. Equal amounts of protein was run on SDS-PAGE and blotted on PVDF membrane (GE Amersham). Following incubation with primary and HRP-conjugated secondary antibodies, the blots were developed using enhanced chemiluminescent substrate (Pierce).

MALDI-based protein annotation. The bands obtained were excised, digested with trypsin (Promega, Madison, WI, USA) and analysed using Applied Biosystems AB 4700 MALDI TOF/TOF mass spectrometer following methodologies by Bienvenut et al. ${ }^{47}$ and Bhattacharya et al. ${ }^{48}$ Briefly, trypsin-digested peptides were spotted with $\alpha$-cyano-4-hydroxycinnamic acid matrix by the sandwich method and mass spectrometry (MS) spectra collected in the positive reflector mode. MS/MS spectra were obtained for the corresponding peptides from the 12 most intense peptides seen in MS spectra. The peak list prepared from raw MS and MS/MS data using GPS explorer V3.6 (Applied Biosystems) was searched against human MSDB database using MASCOT (version 2.0.05; Matrixscience, London, UK) server, and the probability-based MOWSE score was considered to determine hits.

Confocal imaging. Cells were washed thrice in phosphate-buffered saline (PBS). The cells were fixed for $30 \mathrm{~min}$ in $4 \%$ formaldehyde (Merck, Whitehouse Station, NJ, USA). Cells were washed thrice with PBS and permeabilized in $0.2 \%$ 
TritonX100 (Sigma) with 10\% FBS. After $1 \mathrm{~h}$, cells were washed thrice with PBS and incubated with primary antibodies overnight at $4{ }^{\circ} \mathrm{C}$. Cells were washed again and incubated in secondary tagged antibody (Alexa fluor 488 and Alexa fluor 633, Invitrogen, Carlsbad, CA, USA) for $2 \mathrm{~h}$. After washing in PBS, cells were visualized in the confocal mode in a Carl Zeiss LSM 510 Meta microscope (Jena, Germany).

Statistical analysis. Statistical significance of the difference between the different conditions was assessed using a two-tailed Student's $t$-test.

\section{Conflict of Interest}

The authors declare no conflict of interest.

Acknowledgements. We acknowledge J Aster and T Jenuwien for the plasmid constructs of NICD and EZH2, respectively. Anita Roy acknowledges fellowship from CSIR, Government of India. We gratefully acknowledge clinical samples provided by Dr. K Banick and Dr. D Banerjee of MR Bangur Hospital, Kolkata, India, and Ramkrishna Mission Seva Pratisthan, Kolkata, India, respectively. The work was funded by SPGHGD project of DAE, Government of India.

1. Artavanis-Tsakonas S, Rand MD, Lake RJ. Notch signaling: cell fate control and signal integration in development. Science 1999; 284: 770-776.

2. Dumortier $A$, Wilson $A$, MacDonald HR, Radtke F. Paradigms of notch signaling in mammals. Int J Hematol 2005; 82: 277-284.

3. Gray GE, Mann RS, Mitsiadis E, Henrique D, Carcangiu ML, Banks A et al. Human ligands of the Notch receptor. Am J Pathol 1999; 154: 785-794.

4. Shimizu K, Chiba S, Hosoya N, Kumano K, Saito T, Kurokawa M et al. Binding of Delta1, Jagged1, and Jagged2 to Notch2 rapidly induces cleavage, nuclear translocation, and hyperphosphorylation of Notch2. Mol Cell Biol 2000; 20: 6913-6922.

5. Schroeter EH, Kisslinger JA, Kopan R. Notch-1 signalling requires ligand-induced proteolytic release of intracellular domain. Nature 1998; 393: 382-386.

6. Amsen D, Antov A, Jankovic D, Sher A, Radtke F, Souabni A et al. Direct regulation of Gata3 expression determines the $T$ helper differentiation potential of Notch. Immunity 2007; 27: 89-99.

7. Radtke F, Wilson A, MacDonald HR. Notch signaling in T- and B-cell development. Curr Opin Immunol 2004; 16: 174-179.

8. Saito T, Chiba S, Ichikawa M, Kunisato A, Asai T, Shimizu K et al. Notch2 is preferentially expressed in mature $B$ cells and indispensable for marginal zone B lineage development. Immunity 2003; 18: 675-685.

9. Sugimoto A, Yamamoto M, Suzuki M, Inoue T, Nakamura S, Motoda R et al. Delta-4 Notch ligand promotes erythroid differentiation of human umbilical cord blood CD34 + cells. Exp Hematol 2006; 34: 424-432.

10. Mercher T, Cornejo MG, Sears C, Kindler T, Moore SA, Maillard I et al. Notch signaling specifies megakaryocyte development from hematopoietic stem cells. Cell Stem Cell 2008; 3: $314-326$.

11. Cornejo MG, Mabialah V, Sykes SM, Khandan T, Lo Celso C, Lopez CK et al. Crosstalk between $\mathrm{NOTCH}$ and AKT signaling during murine megakaryocyte lineage specification. Blood 2011; 118: 1264-1273.

12. Watts KL, Delaney C, Humphries RK, Bernstein ID, Kiem HP. Combination of HOXB4 and Delta-1 ligand improves expansion of cord blood cells. Blood 2010; 116: 5859-5866.

13. Lam LT, Ronchini C, Norton J, Capobianco AJ, Bresnick EH. Suppression of erythroid but not megakaryocytic differentiation of human K562 erythroleukemic cells by notch-1. J Biol Chem 2000; 275: 19676-19684.

14. Ishiko E, Matsumura I, Ezoe S, Gale K, Ishiko J, Satoh $Y$ et al. Notch signals inhibit the development of erythroid/megakaryocytic cells by suppressing GATA-1 activity through the induction of HES1. J Biol Chem 2005; 280: 4929-4939.

15. Poirault-Chassac S, Six E, Catelain C, Lavergne M, Villeval JL, Vainchenker W et al. Notch/ Delta4 signaling inhibits human megakaryocytic terminal differentiation. Blood 2010; 116: 5670-5678.

16. Meurette O, Stylianou S, Rock R, Collu GM, Gilmore AP, Brennan K. Notch activation induces Akt signaling via an autocrine loop to prevent apoptosis in breast epithelial cells. Cancer Res 2009; 69: 5015-5022.

17. Calzavara E, Chiaramonte R, Cesana D, Basile A, Sherbet GV, Comi P. Reciprocal regulation of Notch and PI3K/Akt signalling in T-ALL cells in vitro. J Cell Biochem 2008, 103: $1405-1412$

18. Nakao T, Geddis AE, Fox NE, Kaushansky K. PI3K/Akt/FOXO3a pathway contributes to thrombopoietin-induced proliferation of primary megakaryocytes in vitro and in vivo via modulation of p27(Kip1). Cell Cycle 2008; 7: 257-266.

19. Tanaka M, Kirito K, Kashii $\mathrm{Y}$, Uchida M, Watanabe $\mathrm{T}$, Endo $\mathrm{H}$ et al. Forkhead family transcription factor FKHRL1 is expressed in human megakaryocytes. Regulation of cell cycling as a downstream molecule of thrombopoietin signaling. J Biol Chem 2001; 276: 15082-15089.
20. Guerriero R, Parolini I, Testa U, Samoggia P, Petrucci E, Sargiacomo M et al. Inhibition of TPO-induced MEK or mTOR activity induces opposite effects on the ploidy of human differentiating megakaryocytes. J Cell Sci 2006; 119: 744-752.

21. Beaulieu LM, Lin E, Morin KM, Tanriverdi K, Freedman JE. Regulatory effects of TLR2 on megakaryocytic cell function. Blood 2011; 117: 5963-5974

22. Ye JY, Chan GC, Qiao L, Lian Q, Meng FY, Luo XQ et al. Platelet-derived growth factor enhances platelet recovery in a murine model of radiation-induced thrombocytopenia and reduces apoptosis in megakaryocytes via its receptors and the PI3-k/Akt pathway. Haematologica 2010; 95: 1745-1753.

23. Ringrose L, Paro R. Epigenetic regulation of cellular memory by the Polycomb and Trithorax group proteins. Annu Rev Genet 2004; 38: 413-443.

24. Su IH, Basavaraj A, Krutchinsky AN, Hobert O, Ullrich A, Chait BT et al. Ezh2 controls B cell development through histone $\mathrm{H} 3$ methylation and Igh rearrangement. Nat Immunol 2003; 4: 124-131.

25. Visser HP, Gunster MJ, Kluin-Nelemans HC, Manders EM, Raaphorst FM, Meijer CJ et al. The Polycomb group protein EZH2 is upregulated in proliferating, cultured human mantle cell lymphoma. Br J Haematol 2001; 112: 950-958.

26. Su IH, Dobenecker MW, Dickinson E, Oser M, Basavaraj A, Marqueron R et al. Polycomb group protein ezh2 controls actin polymerization and cell signaling. Cell 2005 121: 425-436.

27. Jacquelin B, Kortulewski T, Vaigot P, Pawlik A, Gruel G, Alibert $O$ et al. Novel pathway for megakaryocyte production after in vivo conditional eradication of integrin alphallb-expressing cells. Blood 2005; 106: 1965-1974.

28. Nikoloski G, Langemeijer SM, Kuiper RP, Knops R, Massop M, Tonnissen ER et al. Somatic mutations of the histone methyltransferase gene EZH2 in myelodysplastic syndromes. Nat Genet 2010; 42: 665-667.

29. Mochizuki-Kashio M, Mishima Y, Miyagi S, Negishi M, Saraya A, Konuma T et al. Dependency on the polycomb gene Ezh2 distinguishes fetal from adult hematopoietic stem cells. Blood 2011; 118: 6553-6561

30. Huang Z, Dore LC, Li Z, Orkin SH, Feng G, Lin S et al. GATA-2 reinforces megakaryocyte development in the absence of GATA-1. Mol Cell Biol 2009; 29: 5168-5180.

31. Hobert $\mathrm{O}$, Jallal B, Ullrich A. Interaction of Vav with ENX-1, a putative transcriptional regulator of homeobox gene expression. Mol Cell Biol 1996; 16: 3066-3073.

32. Yang N, Higuchi O, Ohashi K, Nagata K, Wada A, Kangawa K et al. Cofilin phosphorylation by LIM-kinase 1 and its role in Rac-mediated actin reorganization. Nature 1998; 393 809-812.

33. Shivdasani RA, Fujiwara $Y$, McDevitt MA, Orkin SH. A lineage-selective knockout establishes the critical role of transcription factor GATA-1 in megakaryocyte growth and platelet development. EMBO J 1997; 16: 3965-3973.

34. Gutierrez L, Tsukamoto S, Suzuki M, Yamamoto-Mukai H, Yamamoto M, Philipsen S et al. Ablation of Gata1 in adult mice results in aplastic crisis, revealing its essential role in steady-state and stress erythropoiesis. Blood 2008; 111: 4375-4385.

35. Shivdasani RA, Rosenblatt MF, Zucker-Franklin D, Jackson CW, Hunt P, Saris CJ et al. Transcription factor NF-E2 is required for platelet formation independent of the actions of thrombopoietin/MGDF in megakaryocyte development. Cell 1995; 81: 695-704.

36. Starck J, Weiss-Gayet M, Gonnet C, Guyot B, Vicat JM, Morle F. Inducible Fli-1 gene deletion in adult mice modifies several myeloid lineage commitment decisions and accelerates proliferation arrest and terminal erythrocytic differentiation. Blood 2010; 116 4795-4805.

37. Shivdasani RA, Orkin SH. Erythropoiesis and globin gene expression in mice lacking the transcription factor NF-E2. Proc Natl Acad Sci USA 1995; 92: 8690-8694.

38. Fujiwara $Y$, Browne $C P$, Cunniff $K$, Goff SC, Orkin SH. Arrested development of embryonic red cell precursors in mouse embryos lacking transcription factor GATA-1. Proc Natl Acad Sci USA 1996; 93: 12355-12358.

39. Gao Z, Huang Z, Olivey HE, Gurbuxani S, Crispino JD, Svensson EC. FOG-1-mediated recruitment of NuRD is required for cell lineage re-enforcement during haematopoiesis. EMBO J 2010; 29: 457-468.

40. Cardoso C, Mignon C, Hetet G, Grandchamps B, Fontes M, Colleaux L. The human EZH2 gene: genomic organisation and revised mapping in $7 q 35$ within the critical region for malignant myeloid disorders. Eur J Hum Genet 2000; 8: 174-180.

41. Essex DW, Chen K, Swiatkowska M. Localization of protein disulfide isomerase to the external surface of the platelet plasma membrane. Blood 1995; 86: 2168-2173.

42. Essex DW, Li M. Protein disulphide isomerase mediates platelet aggregation and secretion. Br J Haematol 1999; 104: 448-454.

43. Bryant RJ, Winder SJ, Cross SS, Hamdy FC, Cunliffe VT. The Polycomb Group protein EZH2 regulates actin polymerization in human prostate cancer cells. Prostate 2008; 68 : 255-263.

44. Cha TL, Zhou BP, Xia W, Wu Y, Yang CC, Chen CT et al. Akt-mediated phosphorylation of EZH2 suppresses methylation of lysine 27 in histone H3. Science 2005; 310: 306-310.

45. Chen TW, Hwang SM, Chu IM, Hsu SC, Hsieh TB, Yao CL. Characterization and transplantation of induced megakaryocytes from hematopoietic stem cells for rapid platelet recovery by a two-step serum-free procedure. Exp Hematol 2009; 37: 1330-1339; e1335.

46. Chen $\mathrm{H}$, Tu SW, Hsieh JT. Down-regulation of human DAB2IP gene expression mediated by polycomb Ezh2 complex and histone deacetylase in prostate cancer. J Biol Chem 2005 280: 22437-22444. 
47. Bienvenut WV, Deon C, Pasquarello C, Campbell JM, Sanchez JC, Vestal ML et al. Matrixassisted laser desorption/ionization-tandem mass spectrometry with high resolution and sensitivity for identification and characterization of proteins. Proteomics 2002; 2: 868-876.

48. Bhattacharya D, Mukhopadhyay D, Chakrabarti A. Hemoglobin depletion from red blood cell cytosol reveals new proteins in 2-D gel-based proteomics study. Proteomics Clin Appl 2007; 1: 561-564.
Cell Death and Disease is an open-access journal SOMERILHIS RESERVED
licensed under the Creative Commons Attribution-NonCommercial-No Derivative Works 3.0 Unported License. To view a copy of this license, visit http://creativecommons.org/licenses/by-nc-nd/3.0/

Supplementary Information accompanies the paper on Cell Death and Disease website (http://www.nature.com/cddis) 